Invited Paper

\title{
Huge Capacity Fiber-Optic Sensing Network Based on Ultra-Weak Draw Tower Gratings
}

\author{
Minghong YANG*, Wei BAI, Huiyong GUO, Hongqiao WEN, Haihu YU, \\ and Desheng JIANG
}

National Engineering Laboratory for Fiber Optic Sensing Technology, Wuhan University of Technology, Wuhan, 430070, China

${ }^{*}$ Corresponding author: Minghong YANG $\quad$ E-mail: minghong.yang@whut.edu.cn

\begin{abstract}
This paper reviews the work on huge capacity fiber-optic sensing network based on ultra-weak draw tower gratings developed at the National Engineering Laboratory for Fiber Optic Sensing Technology (NEL-FOST), Wuhan University of Technology, China. A versatile drawing tower grating sensor network based on ultra-weak fiber Bragg gratings (FBGs) is firstly proposed and demonstrated. The sensing network is interrogated with time- and wavelength-division multiplexing method, which is very promising for the large-scale sensing network.
\end{abstract}

Keywords: Ultra-weak FBG; optical fiber sensors; sensing network

Citation: Minghong YANG, Wei BAI, Huiyong GUO, Hongqiao WEN, Haihu YU, and Desheng JIANG, "Huge Capacity Fiber-Optic Sensing Network Based on Ultra-Weak Draw Tower Gratings," Photonic Sensors, 2016, 6(1): 26-41.

\section{Introduction}

Fiber Bragg grating (FBG) sensors network [1, 2] has attracted considerable interests for quasidistributed sensing. Wavelength-division multiplexing (WDM) is a popular scheme because of its intuitionistic wavelength demodulation method. However, limited by the bandwidth of broadband light sources, often less than $100 \mathrm{~nm}$, only tens of sensors can be multiplexed in one fiber. Childers et al. experimentally multiplexed $800 \mathrm{FBG}$ sensors in a single array by using an optical frequency domain reflectometry (OFDR) scheme [3], but the maximum fiber span is limited by the coherence length of the tunable laser and polarization fading in the light interference. Time-division multiplexing (TDM), distinguishing the FBG sensors with an identical wavelength by detecting the different time delay between reflected pulses along the fiber, has substantial potential for increasing the number of the multiplexing sensors [4-7]. Several TDM schemes with a resonant cavity were reported $[8,9]$. Notably, the required interrogated sensors of these schemes are traditional FBGs with reflectivity $\geq-20 \mathrm{~dB}$, where the multiplexing capacity is seriously limited by crosstalks. Although the multiplexing capacity can be improved to some extent by using the hybrid scheme, such as WDM+TDM, these technologies are limited by the bandwidth and transmission loss. To obtain lower crosstalks and larger multiplexing capability, it is a great choice to use ultra-weak FBGs. Wang et al. experimentally demonstrated 1000 ultra-weak FBG (peak reflectivity $\leq-37 \mathrm{~dB}$ ) by a serial TDM sensor network [10]. However, it 
was a relative long interval to obtain the result of one special FBG sensor, over $10 \mathrm{~s}$, by reconstructing the reflection spectrum of each FBG during the entire scanning period in time domain.

In this paper, a new approach of on-line growing draw tower grating (DTG) and their large-scale sensing network is proposed and demonstrated, and a large-scale sensor network with wavelength scanning time division multiplexed (WSTDM) ultra-weak FBGs (reflectivity of about $-40 \mathrm{~dB}$ ) is realized. The novel ultra-weak DTG technology platform will open new possibility for further application of the large-scale FBG network with thousands of sensing elements. Furthermore, since the ultra-weak reflectance signal is still higher than the Brilloum signal, it can be very promising as a long distance sensing network with a faster response and a more precise positioning capability.

\section{Ultra-weak draw tower grating fabrication}

An on-line writing weak FBG array is the process that $\mathrm{FBGs}$ are directly inscribed into the fiber during drawing. In this case, FBG writing must be done before the fiber coating application, since the fiber coating is usually not transparent to ultro video (UV) light. In the traditional FBG preparation, the fiber has to be decoated before writing FBGs and recoated after the grating exposure. The conventional process is not only difficult to handle, but also it degrades fiber strength, which does damage to its engineering application. However, draw-tower FBGs can overcome these disadvantages, due to the simple operation and high mechanical stability [11, 12]. Furthermore, such a method can produce FBG sensor arrays without fiber fusion, which cannot be avoided in the traditional FBG array construction. No fiber fusion is importance because fusion loss greatly depresses the multiplexing capacity of a weak FBG array. These advantages therefore prompt researchers to begin the research of draw-tower FBGs early [13, 14].
The multiplexing capacity of the traditional FBG sensor arrays generally limits to dozens of FBGs, even though various multiplexing methods are used, e.g., wavelength division multiplexing and time division multiplexing [15-17]. Recently, some investigations reported that identical weak FBG array could greatly improve the multiplexing capacity and sensing distance due to its narrow bandwidth and weak reflection characteristics [18-20]. However, these bright characteristics require that weak FBG array itself has a good uniformity, especially good wavelength uniformity. The draw-tower grating technique is a good choice for preparing identical and weak FBG arrays.

Various factors of central wavelength separation including fluctuations of drawing tension and core temperature were mentioned [13]. However, by detecting the surface temperature of the bare fiber, we found that the core temperature of the bare fiber at the writing spot was stable in the stable drawing state. Nowadays, some draw-tower FBGs are already commercially available [21], however their wavelength accuracy of $\leq 0.4 \mathrm{~nm}$ did not meet the requirements for high quality FBG arrays. In their FBG writing platform, the interference fringes used for writing FBGs were easily influenced by air flow due to a long optical path of the Talbot interferometer [22]. Moreover, tiny changes in the angle and position of two reflecting mirrors would decrease the grating wavelength accuracy. Therefore, even if the phase mask technique was used in their FBG writing platform, the grating wavelength accuracy was not enough. In our experiment, the triangle interference area of the phase mask was directly used for on-line writing identical FBG arrays to depress the effect of the interferometer.

For on-line writing weak FBG arrays, we employed one production-type draw tower as shown in Fig. 1, which could operate at speeds ranging from $3 \mathrm{~m} / \mathrm{min}$ to $200 \mathrm{~m} / \mathrm{min}$ for a bare fiber diameter of $125 \mu \mathrm{m}$. The drawing speed and drawing tension could be automatically controlled and displayed. A 
line-narrowed ArF excimer laser (OptoSystems CL5300) with a beam size of $4 \mathrm{~mm} \times 12 \mathrm{~mm}$, pulse width of $10 \mathrm{~ns}$, maximum pulse energy of $40 \mathrm{~mJ}$, and maximum laser repetition rate of $300 \mathrm{~Hz}$ was used in the FBG writing platform. The reflective spectra of FBGs were gained by one optical analyzer (YOKOGAWA-AQ6370B) with one homemade light emitting diode (LED) source at $1.3 \mu \mathrm{m}$ wavelength band. The OTDR spectra of weak FBG array were acquired by an optical time domain reflectometer (OTDR, YOKOGAWA-AQ7260).

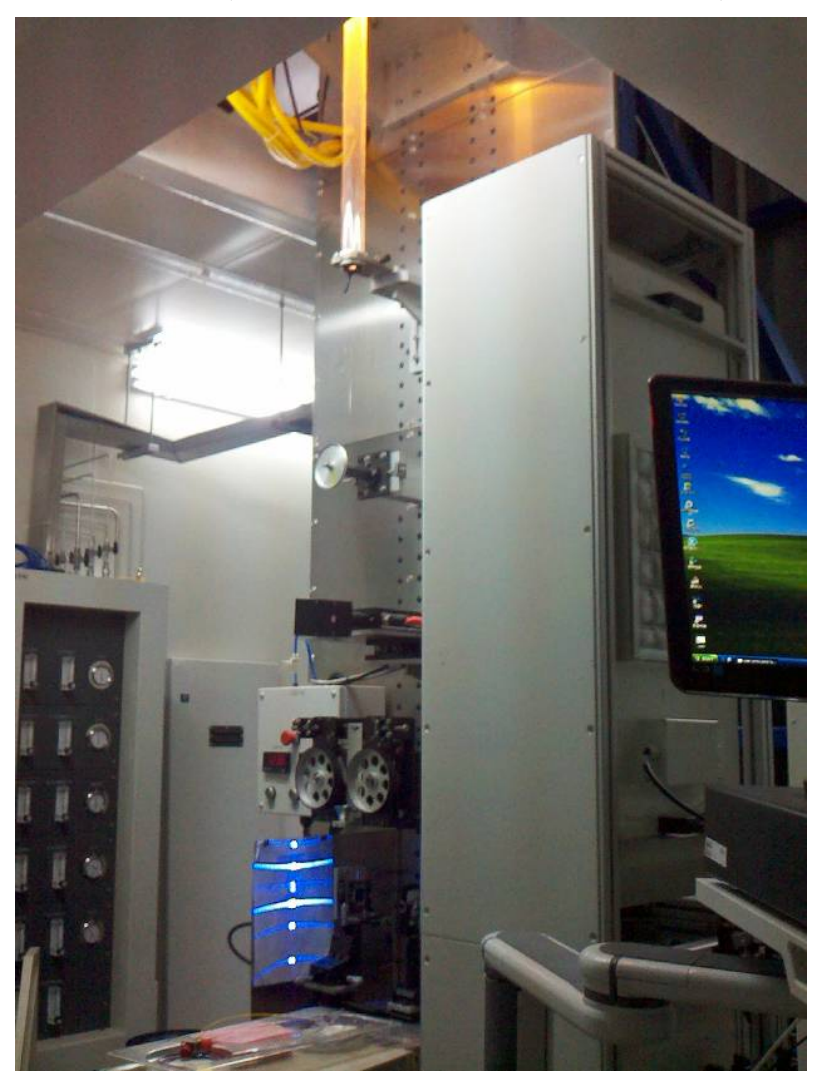

Fig. 1 Production-type draw-tower grating system.

FBG was written by the phase mask method which used periodic interference fringes of the \pm 1 st diffraction light to irradiate photosensitive fiber and hence periodically change the refractive index of fiber core. In this method, the interference fringes were determined by the structure of phase mask, and not influenced by light source coherence, which ensured a good wavelength consistency of FBG arrays. The laser beam was as possible as large to ensure a uniform energy density in a wider writing spot. Additionally, FBG writing platform was mounted on the draw tower near the first coating to weaken fiber vibrating, as shown in Fig. 2. In the experiment, draw-tower FBG arrays were generally carried out under the relatively stable pulse energy of $25 \mathrm{~mJ}$. The laser beam was focused from $4 \mathrm{~mm} \times$ $12 \mathrm{~mm}$ to a line $0.7 \mathrm{~mm} \times 10 \mathrm{~mm}$ using three cylindrical lenses. The distance between the phase mask and bare fiber was controlled at $0.50 \mathrm{~mm}$.

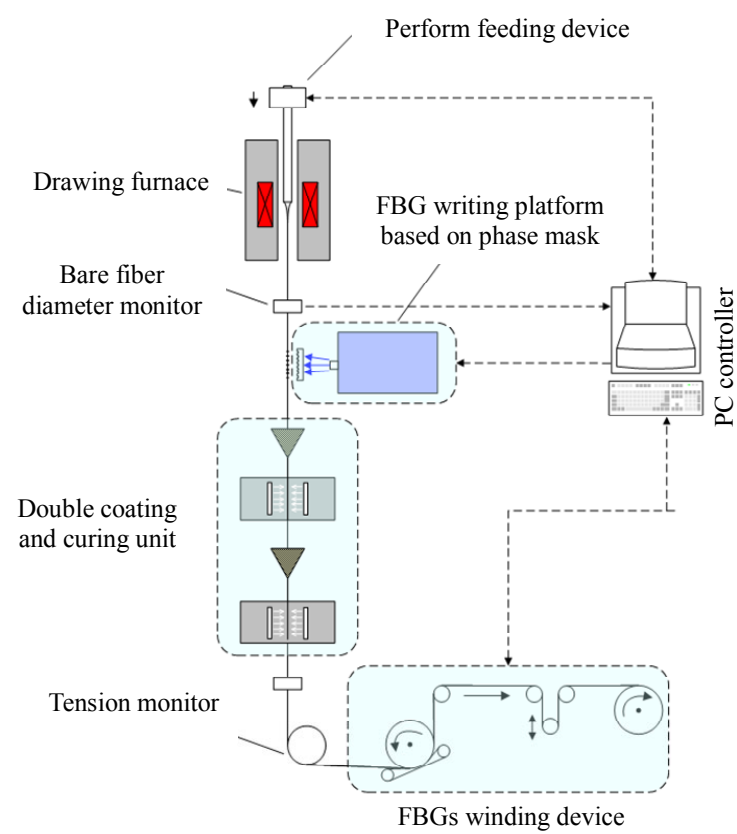

Fig. 2 Diagram of on-line writing FBG system.

The draw-tower FBG array required the fiber with a high single-pulse photosensitivity, since a single laser pulse used here had limited energy and irradiating time. Previous work reported that co-doping $\mathrm{Ge} / \mathrm{B}$ could greatly improve the photosensitivity of optical fiber [23]. Here a Ge/B co-doped perform was carried out in the Optical Fiber Research and Development Department, FiberHome Communication Science and Technology Co., Ltd. The obtained fiber with a B-doped concentration of around $10 \mathrm{~mol} \%$ showed an excellent single-pulse photosensitivity and an acceptable attenuation of $2.8 \mathrm{~dB} / \mathrm{km}$ at $1300 \mathrm{~nm}$. 
Compared with the traditional hydrogen-loaded fiber (immersed in 9-Mpa hydrogen for 1 month), the $\mathrm{Ge} / \mathrm{B}$ co-doped fiber showed the 15 -folds grating reflectivity, also $2.1 \%$, in the same condition: the laser pulse energy of $29 \mathrm{~mJ}$ and the beam size of $0.4 \mathrm{~mm} \times 10 \mathrm{~mm}$. The $\mathrm{Ge} / \mathrm{B}$ co-doped fiber meets on-line writing FBG arrays.

Figure 3(a) shows the OTDR trace of the continuous 200 FBGs in Array A. Each FBG was written successfully. Figure 3(b) displays the local enlargement between $0.8 \mathrm{~km}$ and $0.9 \mathrm{~km}$. Therefore, the spatial separation among Array A was fixed at 5 m. At present, the system could fulfill a spatial separation at a millimeter level with the accuracy of $\pm 1 \mathrm{~mm}$.

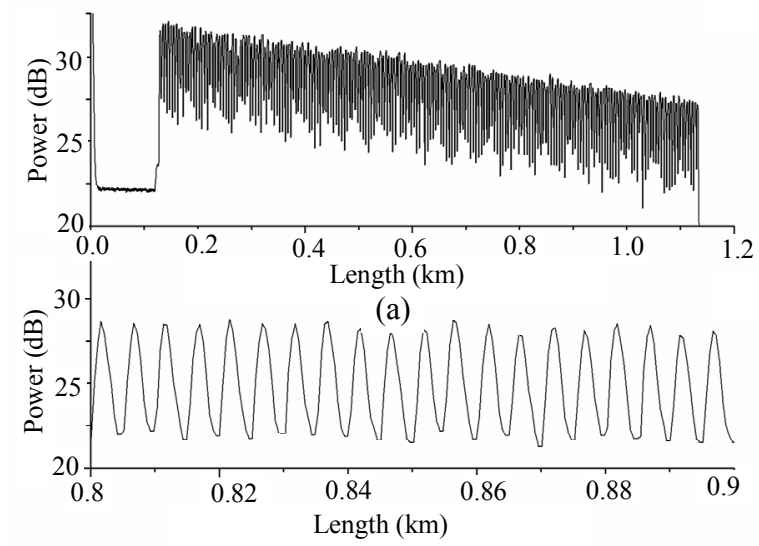

(b)

Fig. 3 OTDR fragment from 200 FBGs of Array A: (a) power evolution of $200 \mathrm{FBG}$ in series and (b) enlargement of 20 FBGs.

Figures 4(a) and 4(b) shows the reflection spectra of Array A (b) and its single FBG (a) without tress. The overlapping spectrum of Array A was almost as same as that of its single FBG. Hence, results indicated that the online-writing FBG array by the phase mask method had a good uniformity of central wavelength.

Effect of the drawing speed on the quality of FBG was firstly studied. Figure 5 shows the reflection spectra of FBGs obtained at different drawing speeds. It can be seen that the shape of reflection spectra was not affected by the drawing speed. In all the reflection spectra, the full width at half maximum (FWHM) and reflection power of the main peak were $0.09 \mathrm{~nm}$ and $\sim 7 \mathrm{pW}$, respectively. This indicated that FBGs could be written perfectly at the drawing speeds of interest. Generally, the drawing tension was large at the high drawing speeds. This would deteriorate the central wavelength uniformity of FBG arrays (Fig. 6). Additionally, stabilizing the drawing process often took more than one hour at the high drawing speed. Therefore, in our experiment, on-line writing identical FBG arrays were done at the lower drawing speed of $\sim 15 \mathrm{~m} / \mathrm{min}$.

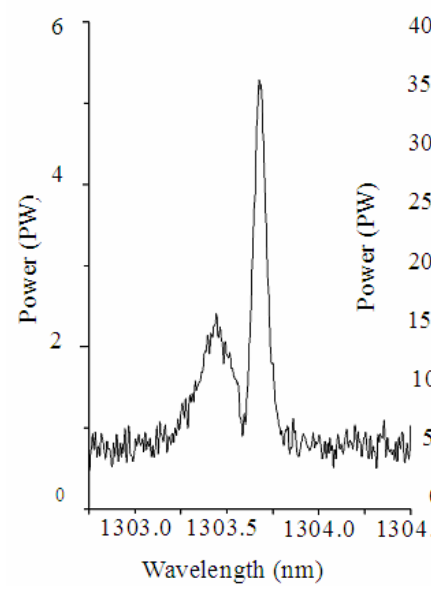

(a)

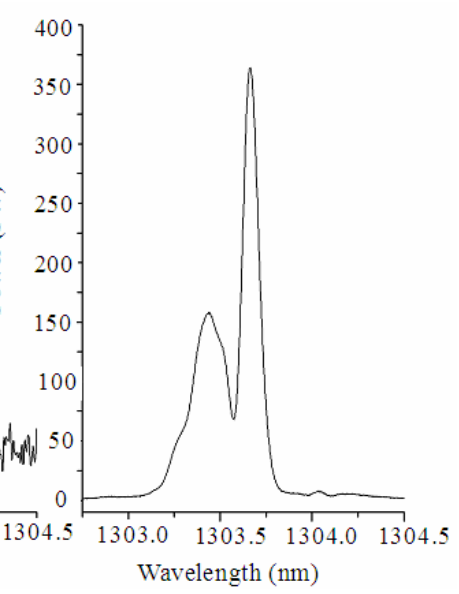

(b)
Fig. 4 Reflection spectra of the weak FBG investigated with the light source power of $2.5 \mathrm{nW}$ at $1303.638 \mathrm{~nm}$ : (a) array of 200 FBGs and (b) single FBG.

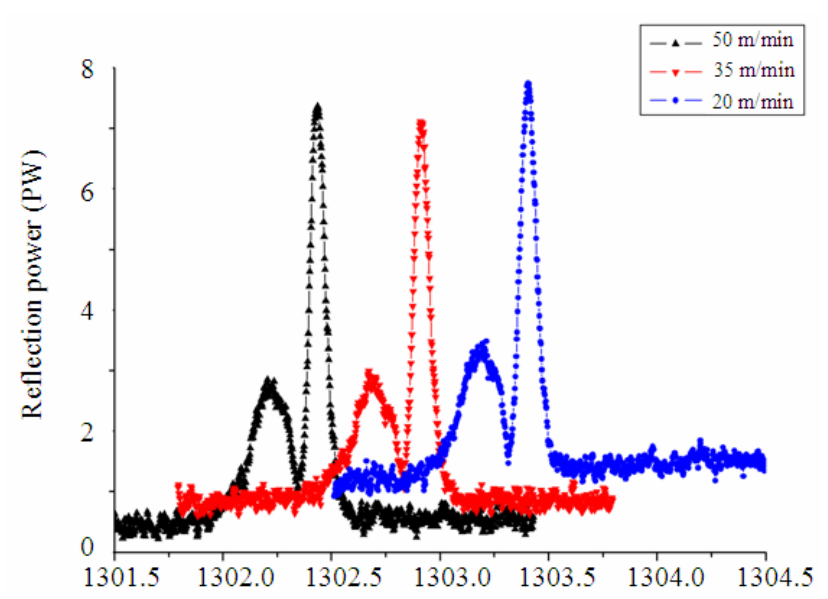

Fig. 5 Reflection spectra of three FBGs written at different speeds. 
The wavelength uniformity of FBG arrays was important, because it was closely related to the data volume of terminal signal demodulation. Although the drawing process reached the stable work state, a small fluctuation of the drawing speed was always present to control the fiber diameter in a qualified range. Hence, the fluctuation of the drawing tension was recorded to follow the change in the drawing speed. We found that the central wavelength drifted reversely with an increase in drawing tension (Fig. 7). This suggested that the quality of FBG arrays was very sensitive to the tiny fluctuation of the drawing speed.

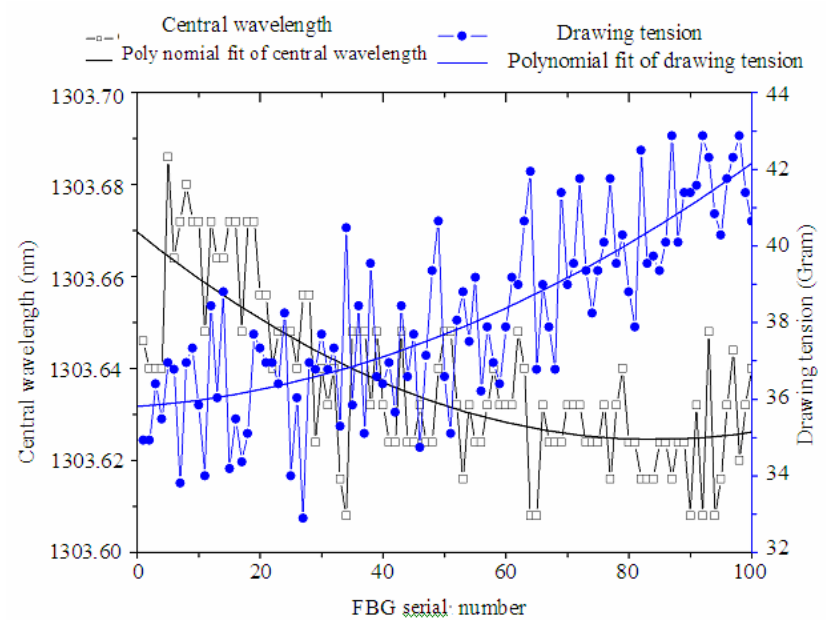

Fig. 6 Central wavelength and drawing tension traces of successive 100 FBGs in Array A.

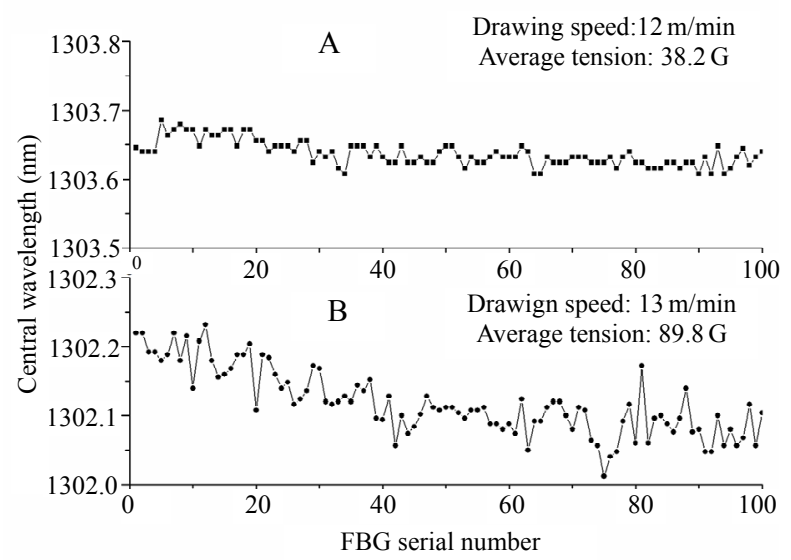

Fig. 7 Central wavelength distributions of successive 100 FBGs in Array A and Array B.

Here the central wavelength uniformity of on-line writing FBG array was investigated under different drawing tension (the drawing tension increased through decreasing the furnace temperature), as shown in Fig. 6. From Fig. 7, we can see that in Array A, the central wavelengths of 100 FBGs vary by $\sim 0.1 \mathrm{~nm}$ from $1303.608 \mathrm{~nm}$ to $1303.686 \mathrm{~nm}$. In Array B, fluctuation of the central wavelength reaches up to $0.25 \mathrm{~nm}$, which covers from $1302.000 \mathrm{~nm}$ to $1302.250 \mathrm{~nm}$. Additionally, the fluctuation of adjacent wavelengths became larger under the large drawing tension. Notably, the change in the drawing speed was small for both the FBG arrays, and hence results suggested that the large drawing tension was not a good condition. Our studies indicated that a stable and proper drawing tension was a critical factor that ensured online-writing FBG array with good wavelength uniformity.

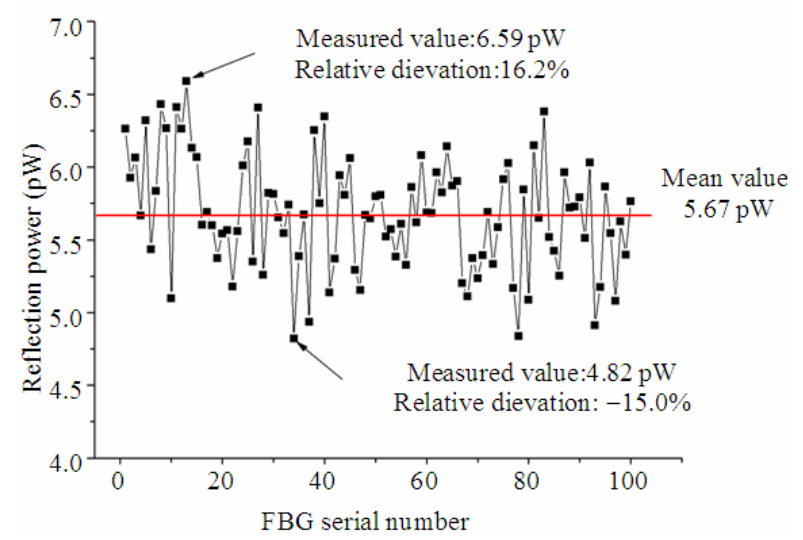

Fig. 8 Single FBG reflection power of 100 FBGs in Array A, investigated with the light source power of $2.5 \mathrm{nW}$ at $1303.638 \mathrm{~nm}$.

To investigate the uniformity of the reflection spectra, single FBG reflection power in Array A was measured successively. Figure 8 shows the reflection power of 100 FBGs in Array A. It could be seen that the relative deviation was about $\pm 16 \%$. During the draw-tower grating preparation, the output energy of laser pulse presented a fluctuation of $\pm 8 \%$. This suggested that effect of fiber vibration was not ignored on the fluctuation of FBG reflection power. Figure 9 exhibited the vibration of bare optical fiber in the exposure area. Since the laser energy density presented Guass distribution in the horizontal 
direction, it became more uneven from the middle to both sides after spot focus. Therefore, the fiber vibration in the beam spot would markedly affect the grating reflectivity.

To decrease the fiber vibration, effects of both the drawing speed and the drawing tension were investigated. When the drawing tension was smaller than 10 grams where the drawing speed was low, we observed that the lateral vibration of optical fiber was obvious. The lateral vibration was greatly improved with increasing the drawing tension up to 30 grams. However, it was difficult to improve greatly the reflectance consistency of FBG array by further decreasing fiber vibration. Other methods for improving the reflectivity uniformity of draw-tower FBG array should be found, e.g., by homogenizing the energy density and by further widening the horizontal dimension of writing spot.

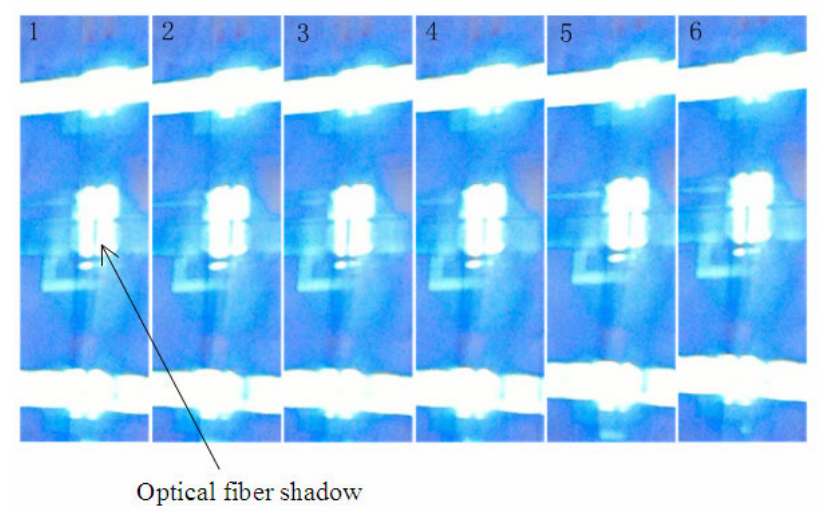

Fig. 9 Six shooting photos of the diffraction spot, taken under the condition: the drawing speed of $12 \mathrm{~m} / \mathrm{min}$, the average drawing tension of 38.2 grams, and laser frequency of $50 \mathrm{~Hz}$.

\section{Interrogation method of the ultra-weak draw tower gratings}

The large capacity sensing network interrogation system with identical ultra-weak FBG is illustrated in Fig. 10. The light from ASE source was modulated to nanosecond pulses. A pulse generator, realized by field programmable gate array (FPGA) logic, driven the first semiconductor optical amplifier $[\mathrm{SOA}(1)]$ functioning as a modulator as well as the first stage optical amplifier. Then the pulses were amplified by the second stage optical amplifier, Erbium-doped fiber amplifier, and launched into a FBG array. The reflected pulses from the FBGs returned to $\mathrm{SOA}(2)$ which acted as a gating device as well as an amplifier. Field programmable gate array (FPGA) generated a time-shifted pulse sequence to activate $\operatorname{SOA}(2)$ to filter and amplify one specific FBG signal while absorbing signals reflected from other FBGs. The pulses reflected by FBGs arrived at $\operatorname{SOA}(2)$ with time delays of:

$$
\tau_{i}=\frac{2 n L_{i}}{c}
$$

where $\tau_{i}$ is the time delay of pulses reflected by $\mathrm{FBG}_{i}, c$ is the speed of light in vacuum, $n$ is the effective refractive index of optical fiber, and $L_{i}$ is the distance of $\mathrm{FBG}_{i}$ from the circulator. When the time-shifted pulse sequence from FPGA have a time shift $\tau_{i}$ the pulse reflected by $\mathrm{FBG}_{i}$ will be amplified each time when they pass through $\mathrm{SOA}(2)$, and the pulses reflected by other FBGs are blocked. By changing delay time through FPGA, each FBG can be addressed separately.

A charge-coupled device (CCD) based demodulator (I-MON 256, Ibson Photonics) was used in the interrogation system to obtain the spectral information of FBGs. The interrogation speed of the system which is shown in (2) was limited by both the response time of CCD $\tau_{\text {res }}$ and the time interval of pulse sequence.

$$
f \leq \min \left(1 / \tau_{d}, 1 / \tau_{\text {res }}\right)
$$

where $\tau_{d} \geq 2 n L / c, L$ is the length of sensing fiber. The response time of CCD could be as low as tens of microseconds. For a sensing fiber less than $10 \mathrm{~km}$ in which 1000 FBGs are serial multiplexed, it takes less than 0.1 second to obtain the reflection wavelengths of all sensors. Once $\operatorname{SOA}(2)$ is turned on, a trigger pulse generated by FPGA will be sent to $\mathrm{CCD}$ to active an spectra sampling. Both $\mathrm{CCD}$ detector and SOAs are controlled by FPGA, so that the spectral sampled by CCD in time delay $\tau_{i}$ can be respond to $\mathrm{FBG}_{i}$. 


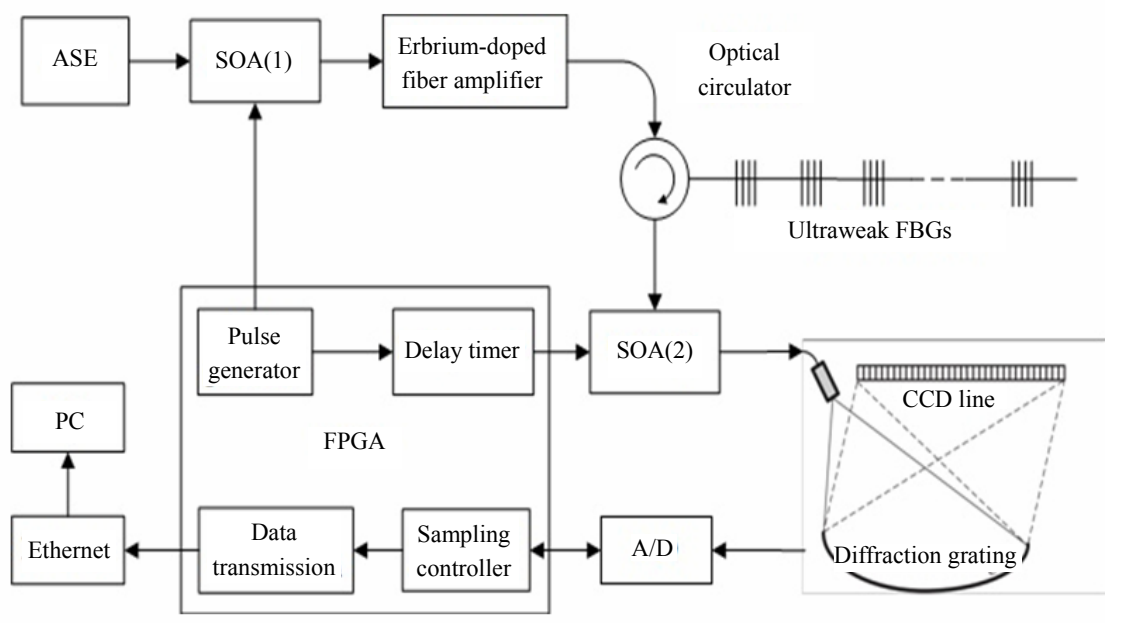

Fig. 10 Illustration of interrogation system.

The reflected spectra of $\mathrm{FBG}_{i}$ need to be reconstructed at different delay time $\tau_{i}$ through peak fitting algorithm because of the limited pixel number of CCD. The average pixel spacing of IMON 256 in wavelength is about $170 \mathrm{pm}$. By using Gaussian peak fitting algorithm, a peak wavelength resolution of $0.5 \mathrm{pm}$ can be achieved. The adjusted Gaussian function is shown in (3):

$$
f\left(\lambda_{i}\right)=A \cdot \exp \left[-\frac{\left(\lambda_{i}-B\right)^{2}}{2 C^{2}}\right]
$$

where $A, B$, and $C$ are the adjusted parameters (amplitude, center and deviation), and $f\left(\lambda_{i}\right)$ is the calculated spectra of $\lambda_{i}$. Gaussian peak fitting is computational intensive, so faster algorithms, such as centroid algorithm, can be used in high-speed interrogation in cost of higher fit noise.

In order to obtain two pulse sequences with time delay precision as high as $1 \mathrm{ps}$, a two-stage time delay controller (coarse tuning and fine tuning) was implemented. Figure 11 shows the procedure. The reference pulse sequence and delayed pulse sequence were named as $1 \#$ and $2 \#$, respectively. The pulse waveform was realized by mapping into block random-access memory (BRAM). The width of pulse was expressed by the continuous digital "1" stored in BRAM, and low level of pulse was corresponding to continuous digital " 0 ". Then the pulse cycle was expressed by the read/write BRAM depth. Output parallel-to-serial logic resources (OSERDESE) were a dedicated parallel-to-serial converter with specific clocking and logic resources in FPGA. If converting the parallel data to serial bit signal of I/O interface by OSERDESE after reading BRAM sequentially, the coarse tuning was completed. Coarse tuning was the first stage delay control unit with the level of accuracy up to $0.625 \mathrm{~ns}$ for Xilinx Virtex7 FPGA.

Programmable output delay (ODELAY) near FPGA ports allowed outgoing signals to be delayed on an individual basis. This was the second stage delay control unit, fine tuning. The tap delay resolution was varied by selecting different FPGA series and different reference clock, generally $78 \mathrm{ps}$ or $52 \mathrm{ps}$. In all 32 taps, adjustable delay can reach $2.496 \mathrm{~ns}$ or $1.664 \mathrm{~ns}$.

The I-MON 256 module has a 256-pixel-InGaAs detector running at a clock frequency of $5 \mathrm{MHz}$ in maximum. Figure 12 shows the system design scheme for spectra sampling. The detector began exposure integral when detecting a high level at a falling edge of CLK, and end exposure integral when detecting a low level at a falling edge of CLK. Pixels data will output serially after 8 CLK.

A/D chip AD9826, 3-Channel 16-bit Operation up to 15 MSPS A/D Converter, works at 1-channel SHA mode timing and converts analog signal to 
16-bit valid pixel data for FPGA. Before InGaAs detector works at normal status, FPGA configures AD9826 by SCLK, SLOAD, and SDATA. CDSCLK2 and ADCCLK output the control clock with $1 \mathrm{MHz}$ and phase difference of 90 degree. The string and conversion will be reached when the high and low byte data are combined. The time measurement data have been received and packaged to PC by Ethernet work after data are cumulated to 256.

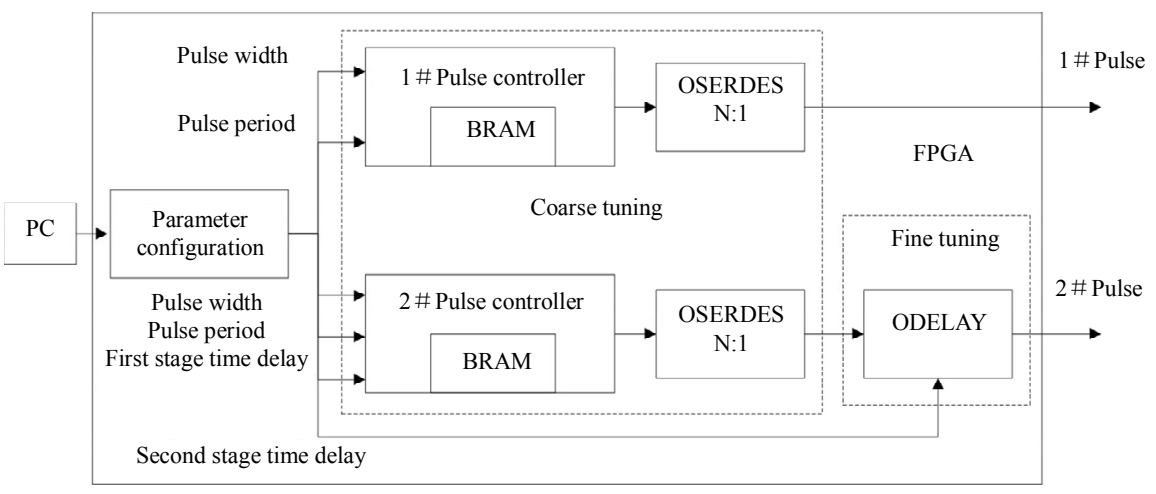

Fig. 11 Modulation pulses generated by FPGA method.

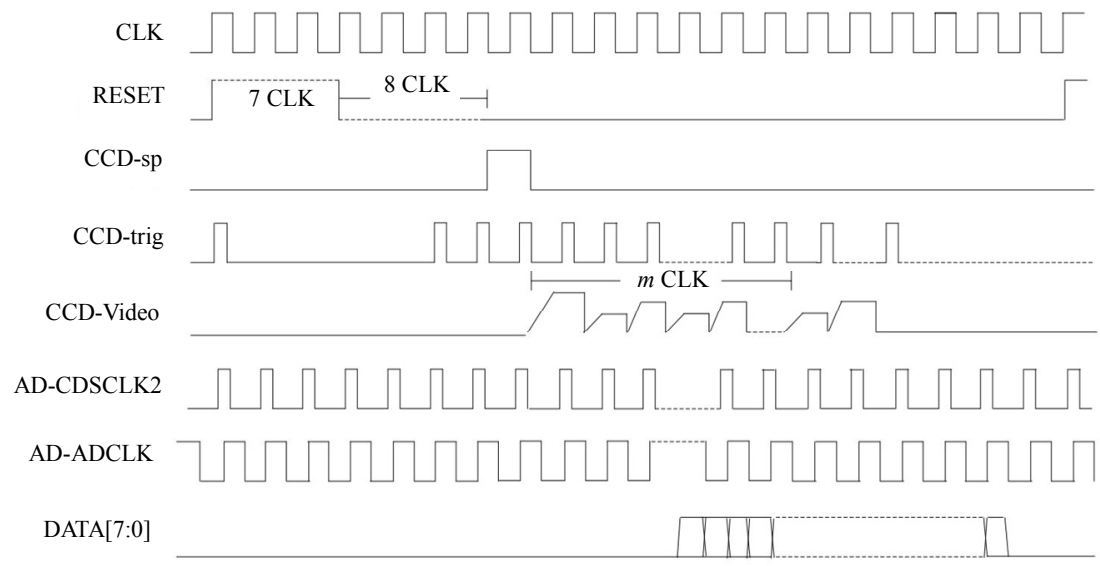

Fig. 12 FPGA timing of InGaAs detector.

The performance of the proposed interrogation system was further verified by demodulating an ultra-weak FBG array with 1009 FBGs and 2.5 meters equidistance between neighbor FBGs. The peak reflectivity of the FBGs was about $-33 \mathrm{~dB}$. The ultra-weak FBG array was fabricated by the on-line writing method [11]. All FBGs in the array were inscribed with a same phase mask so that they have almost the same peak wavelength. FPGA generated a pulse sequence with $20 \mathrm{~ns}$ width and 100 $\mu$ s interval to drive SOA (1). By changing time delay with a step of $1 \mathrm{~ns}$ through delay timer, the reflectance along the sensing fiber was obtained by a
CCD detector. The peaks of the reflectance indicated the locations of FBGs. The normalized reflectance is shown in Fig. 13. The fluctuation of reflectivity was caused by fabrication errors induced during inscribing process.

Figure 14 shows peak wavelengths of all 1009 FBG sensors measured by the interrogation system. Peak wavelengths of the most of FBGs were distributed in the range from $1550.4 \mathrm{~nm}$ to $1550.65 \mathrm{~nm}$. The CCD detector worked at $1000 \mathrm{~Hz}$, and it took about 1 second to complete one interrogation process for a 1009-FBG-array with a distance of about $2.7 \mathrm{~km}$. 

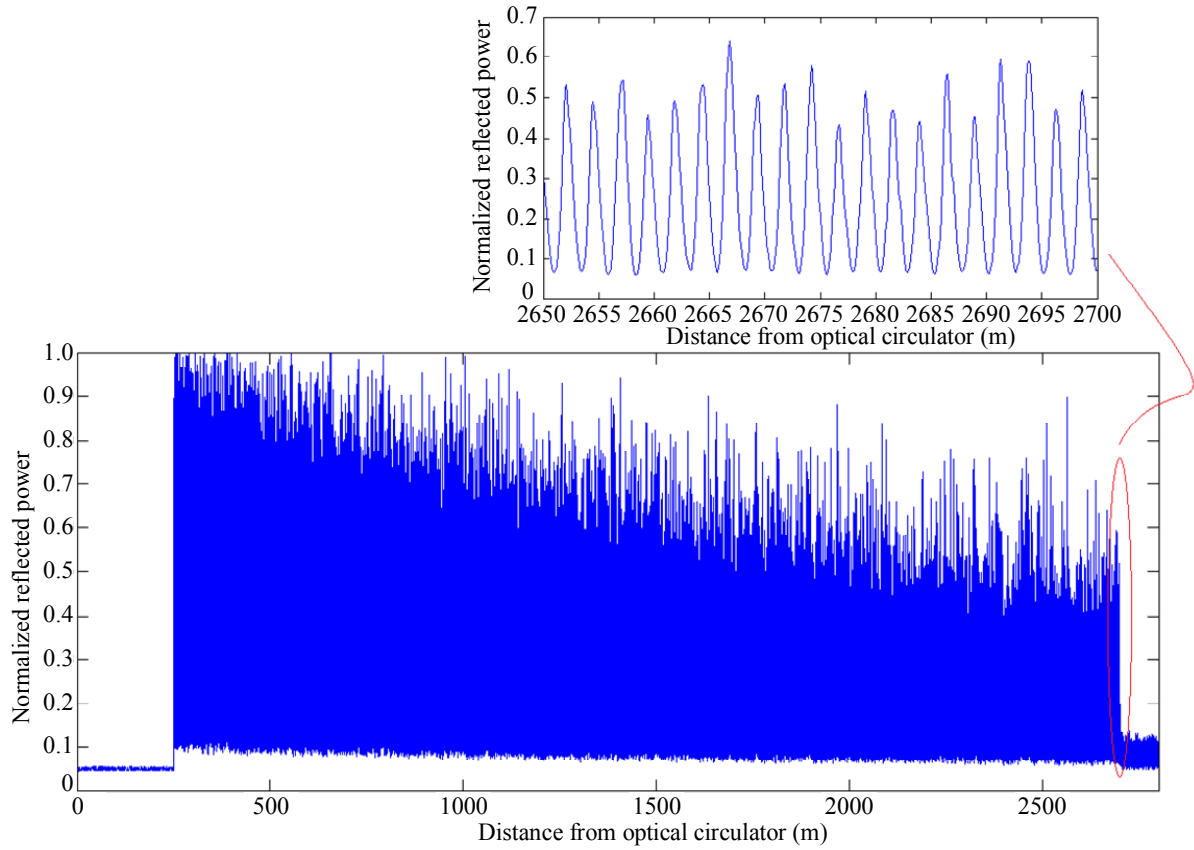

Fig. 13 Reflected power of FBG sensors (reflectivity $=-33 \mathrm{~dB}$ ).

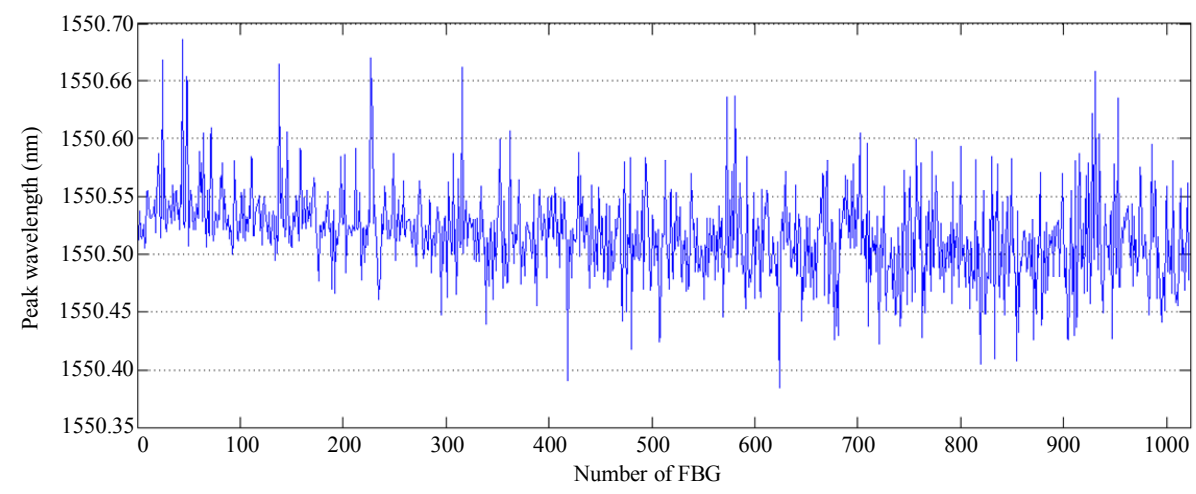

Fig. 14 Peak wavelength of FBG sensors.

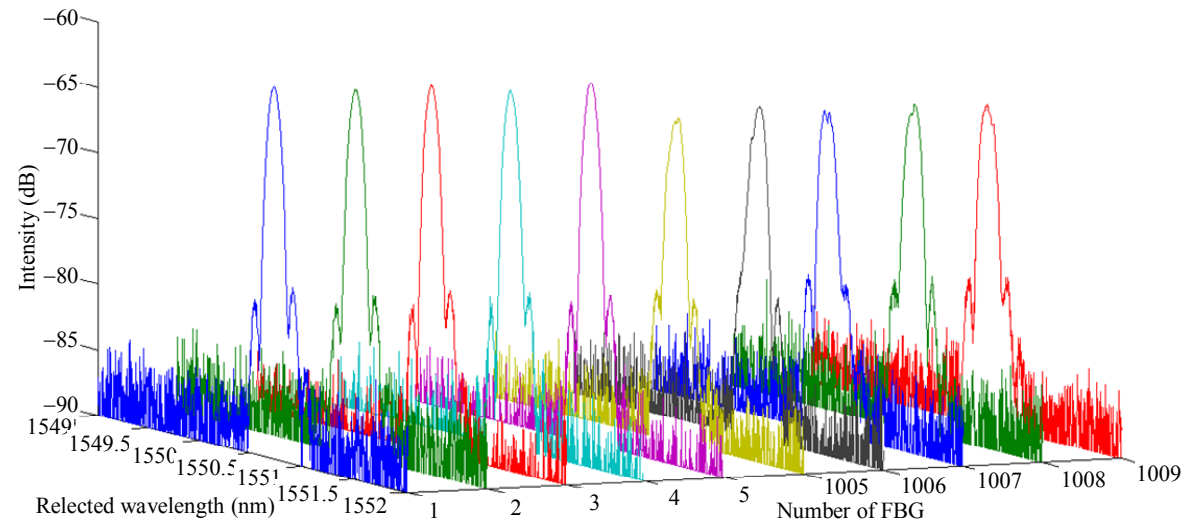

Fig. 15 Reflective spectrum of FBG sensors. 
In order to investigate the spectral distortion induced by the crosstalks, the reflective spectrum of the first and last 5 FBGs of the array were measured by Spectrometer (YOKOGAWA AQ6370B), as shown in Fig. 15, by setting the delay timer to $2517 \mathrm{~ns}, 2543 \mathrm{~ns}, 2566 \mathrm{~ns}, 2592 \mathrm{~ns}, 2616 \mathrm{~ns}, 27716 \mathrm{~ns}$, $27740 \mathrm{~ns}, 27765 \mathrm{~ns}, 27791 \mathrm{~ns}$, and $27815 \mathrm{~ns}$, respectively. The time delay of $2517 \mathrm{~ns}$ for FBG1 indicated there was a bare fiber of about $250 \mathrm{~m}$ between FBG1 and the circulator. The difference of delay time between two adjacent FBGs was about $25 \mathrm{~ns}$. The peak reflectivity of the last 5 FBGs were $-65.294 \mathrm{dBm},-64.619 \mathrm{dBm},-65.423 \mathrm{dBm}$, and $-65.052 \mathrm{dBm},-65.522 \mathrm{dBm}$, respectively, which was $3 \mathrm{~dB}$ less than that of the first 5 FBGs because of multiple reflection crosstalks. There were tiny distortions in the spectrum of the last 5 FBGs which were also caused by multiple reflection crosstalks. The distortion in the end of sensors array will be further reduced by using FBG sensors with even lower reflectivity such as $-40 \mathrm{~dB}$.

To test the impact of the spectral distortion on measurement accuracy, the Bragg wavelength of the sensor array is measured 200 times in the save environment. Based on the data collected from CCD detector, the peak wavelength was calculated by a Gaussian peak fitting method. The measurement accuracy was less than $5 \mathrm{pm}$ for all sensors in the array. Figure 16 shows the standard deviation of the first and last 5 FBGs. The wavelength measurement error $(2 \sigma)$ was limited in $2 \mathrm{pm}$. The impact of spectral distortion caused by crosstalks was negligible.

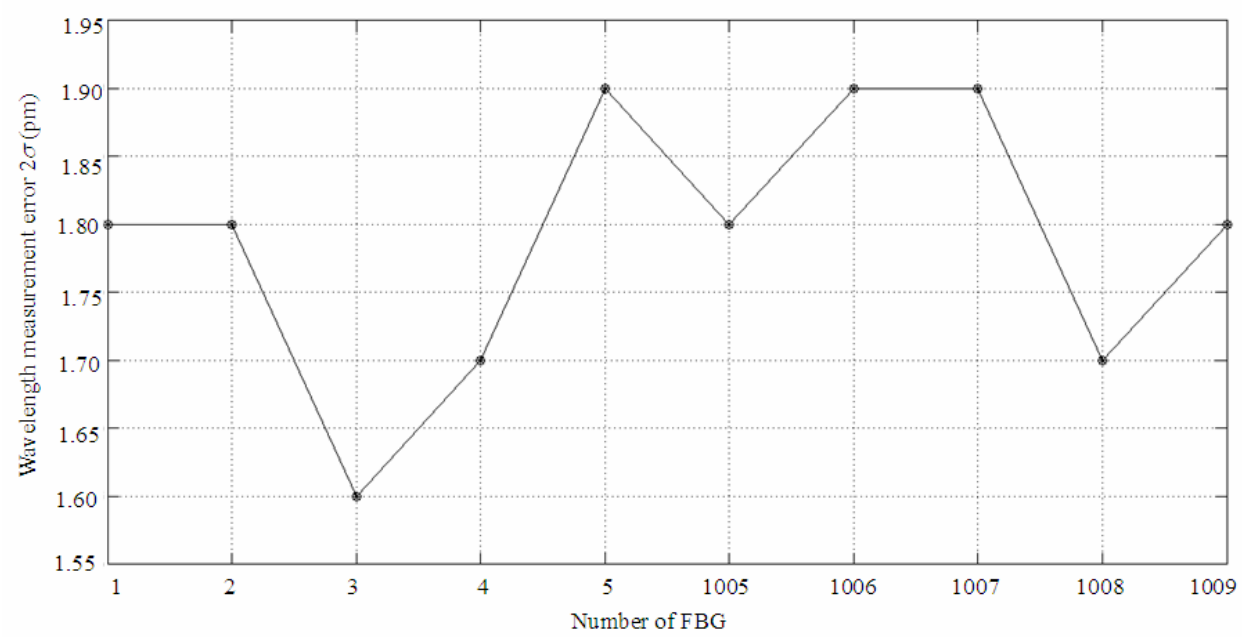

Fig. 16 Measurement error of peak wavelength for FBG1- FBG5 and FBG1005- FBG1009.

\section{Experimental demonstration of fiber- optic sensing network based on ultra- weak FBG}

The performance of the proposed interrogation system was demonstrated by demodulating an ultra-weak 2000-DTGs array with one meter equidistance between neighbored DTGs. The weak DTG array was fabricated by on-line writing method as mentioned above with one phase mask so that they have almost the same peak wavelength. The average reflectivity of the DTG array was about
$-31.5 \mathrm{~dB}$, and all peak wavelengths of the ultra-weak DTG array fell within the range of $1552.075 \mathrm{~nm} \pm$ $0.15 \mathrm{~nm}$, in which those of $95 \%$ DTGs were within the smaller range of $1552.075 \mathrm{~nm} \pm 0.05 \mathrm{~nm}$. Figure 17(a) shows the reflective spectra of 38 representative DTGs in the array interrogated in the forward and reverse directions, respectively, of which serial numbers (in the forward direction) are 1 , $2,3,4,5,6,7,8,9,10,20,30,40,50,60,70,80$, $90,100,200,300,400,500,600,700,800,900$, $1000,1100,1200,1300,1400,1500,1600,1700$, 
1800, 1900, and 2000. The results in the two interrogation indicated that the reflective spectra of the downstream DTGs in the array were distorted severely into the flat-top ones with some sharps and they have different top morphologies from each other. The flatting reason for the reflection peaks of the downstream DTGs is not difficult to understand, which should be attributed to the shadowing effect of upstream DTGs [24, 25]. According to the formula [24]:

$C_{i}(\lambda)=\frac{(i-1)(i-2)}{2} R^{3}(\lambda)(1-R(\lambda))^{2 i-4} I_{0}(\lambda), \quad i \geq 3$.
The one-order crosstalks intensity of the $i$ th DTG is not only related to its location in the array, but also is closely related to the reflectivity of wavelength $\lambda$ of every upstream DTG. Due to the peak wavelength distribution in a certain range, the reflected spectra of the DTG array were dislocated mutually, and as for a certain wavelength $\lambda$, the reflectivity of every upstream DTG may be different; as for different wavelength dots, the one-order crosstalks of the $i$ th will be different, which will give rise to some sharps in the top of the DTG reflection spectra. Due to an ideal

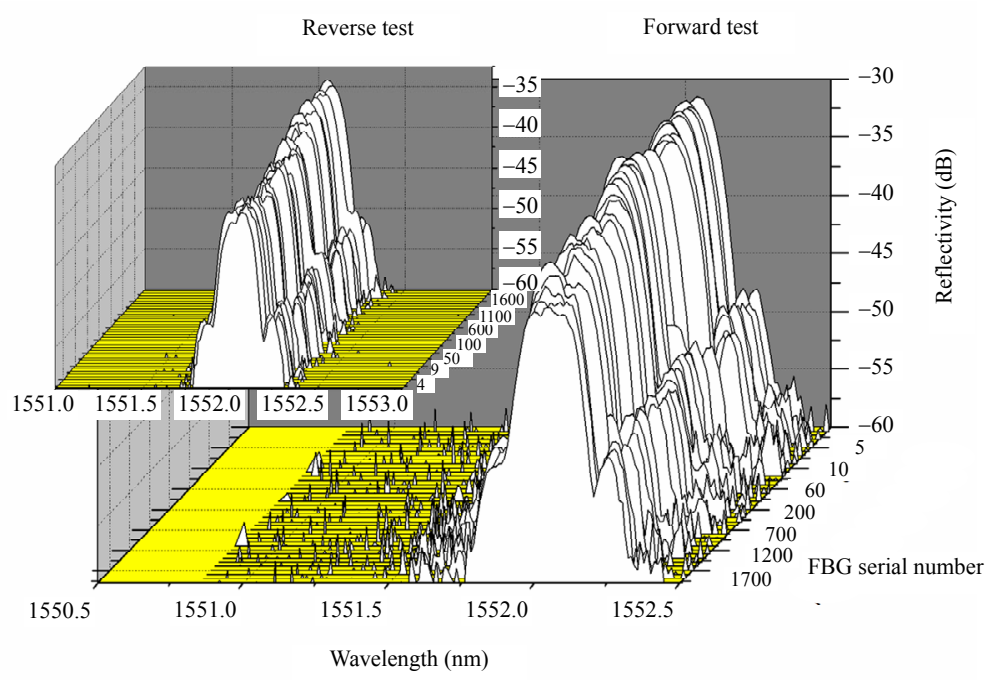

(a)

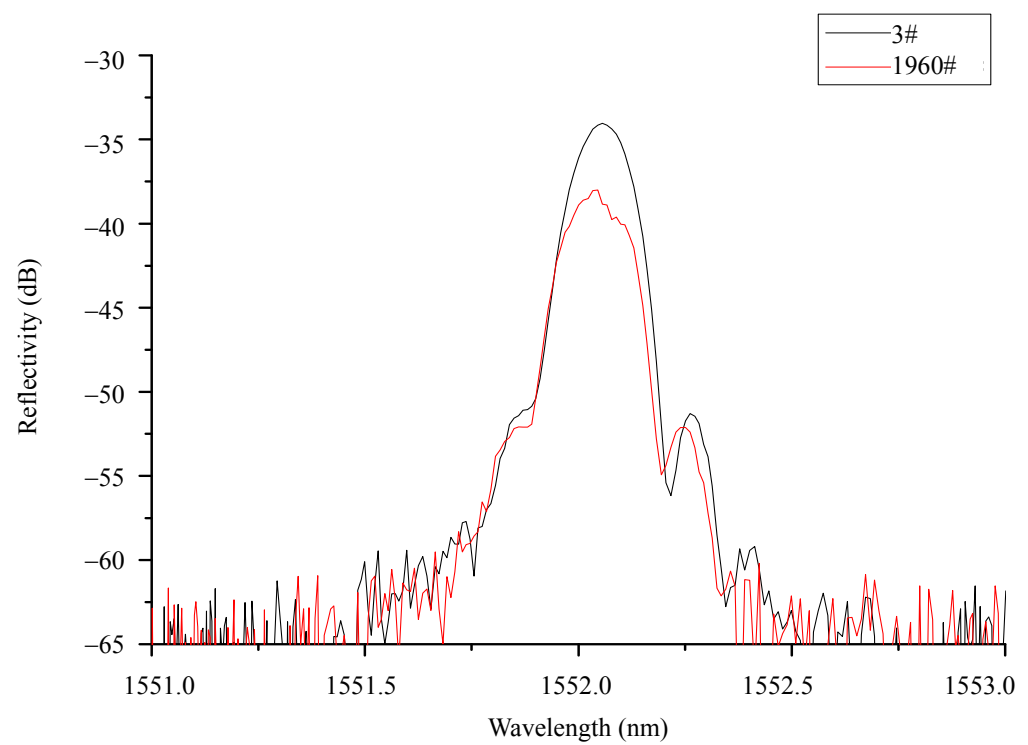

(b) 


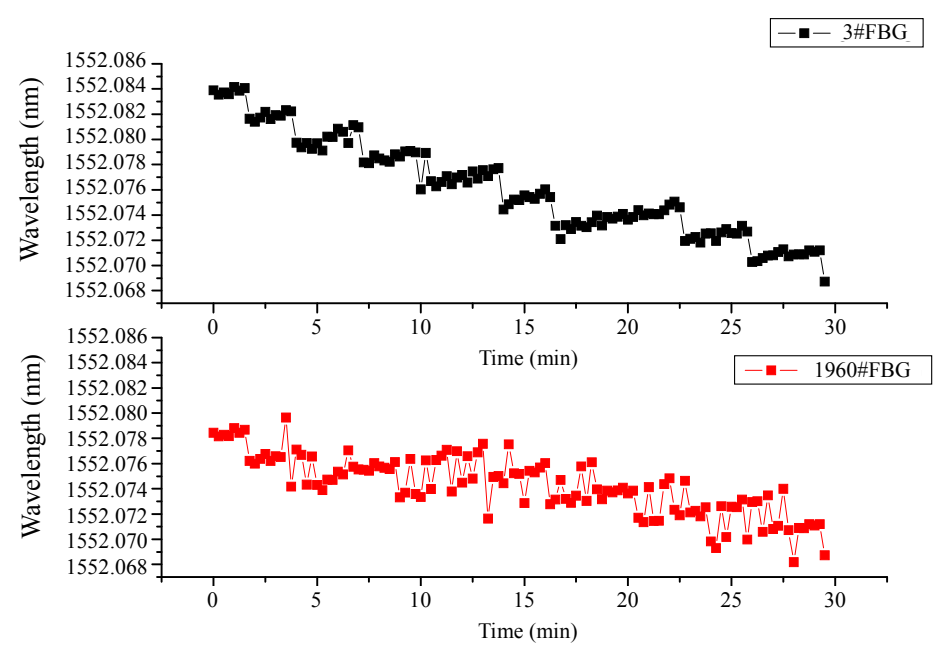

(c)

Fig. 17 Spectra of 1960 DTGs and their peak wavelength variation: (a) reflection spectra of 38 representative DTGs interrogated in the forward and reverse directions, (b) reflection spectra of the 3\# and 1960\# DTGs, and (c) their peak wavelengths interrogation trace over half an hour.

extinction ratio of fiber Bragg grating, the reflectivity of the spectral slope is extremely weak, and so the negative effect of the one-order crosstalks on the spectral slope is ignorable, which can be seen from the symmetrical slopes of all the spectra in Fig. 17(a). Based on the symmetrical slopes, the distorted spectra suffered from signal crosstalks are corrected by Gauss fitting to demodulate the peak wavelengths of the DTG array. The normal reflection spectrum (3rd DTG) and the distorted reflection spectrum (1960th DTG) are shown in Fig. 17(b), and their peak wavelength changes over half an hour were traced [Fig. 17(c)]. Compared with the 3rd DTG, the peak wavelength uncertainty of the 1960th DTG occurred. However, the peak wavelength uncertainty was limited within $\pm 5 \mathrm{pm}$ by the Guass-fitting method. When the peak wavelength was determined by the method of maximum value, its uncertainty can reach up to tens of picometers.

Obviously, if the distorted spectra are used as sensing media in engineer application, measurement accuracy will be worse. It will be crucial for an ideal measurement accuracy to guarantee gaining perfect reflection spectra from all DTGs in the sensing array. The simulation results told that the ultra-weak reflectivity of the DTG array can relieve greatly the signal crosstalks [26]. On the other hand, a bad uniformity on peak wavelengths can also alleviate the crosstalks in the DTG array and guarantee to gain perfect reflection spectra. By adjusting the drawing tension in the DTG preparation process, a DTG array with a whole span of $4400 \mathrm{~m}$, its DTG number of 1418 , and a spacing of $3 \mathrm{~m}$ between two neighboring DTGs was prepared, and its peak wavelengths fluctuated mainly in the range of $1552.945 \mathrm{~nm} \pm 0.2 \mathrm{~nm}$ [shown in Fig. 18(a)]. The DTG array had one average reflectivity of about $-24 \mathrm{~dB}$ originally and had one degraded reflectivity of about $-38 \mathrm{~dB}$ after one $80^{\circ} \mathrm{C}$ isothermal aging time of 2 months. 16 representative DTGs were selected to show their reflection spectra in two cases, and their serial numbers were 1, 100, 200, 300, 400, 500, 600, 700, 800, 900, 1000, 1100, 1200, 1300, 1400 , and 1418. Due to a high average reflectivity of $-24 \mathrm{~dB}$, the reflection spectra of the most DTGs in the wavelength-concentrated area had serverely distorted by the signal crosstalks, and this phenomenon occurred early on the reflection spectrum of the 100\# DTG [Fig. 18(b)]. The peak wavelength reflectivities of $1 \#, 1400 \#$, and 1418\# DTGs are about $-24 \mathrm{~dB},-31 \mathrm{~dB}$, and $-24.5 \mathrm{~dB}$, respectively. Compared with the $1 \#$ DTG, the 
reflectivity of the $1400 \#$ DTG dropped by $7 \mathrm{~dB}$, while only $0.5 \mathrm{~dB}$ for the last 1418 \# DTG whose peak wavelength was drifted out from the wavelength-concentrated area. When the average reflectivity dropped to $-38 \mathrm{~dB}$, all the reflection spectra of the DTGs array appeared to be perfect and for some certain reasons, their wavelength uniformity became worse after the aging process [Fig. 18(c)]. The peak wavelength reflectivities of 1\#, $1400 \#$, and $1418 \#$ DTGs are about $-38 \mathrm{~dB},-40.5 \mathrm{~dB}$, and $-38.9 \mathrm{~dB}$, respectively. Compared with the $1 \#$ DTG, the reflectivity of the 1400 \# DTG dropped by $2.5 \mathrm{~dB}$, while only $0.9 \mathrm{~dB}$ for the last 1418 \# DTG. Experimental results indicated that the reflectivity decline and a worsen wavelength uniformity were beneficial to improve the grating spectra and gain a uniform power distribution in the DTG array, which were very helpful for a high precision detection of the sensor system.

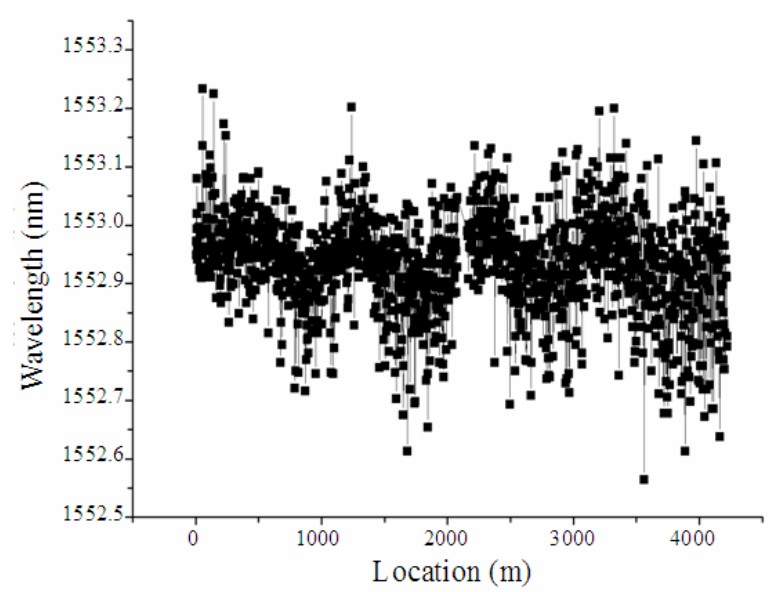

(a)

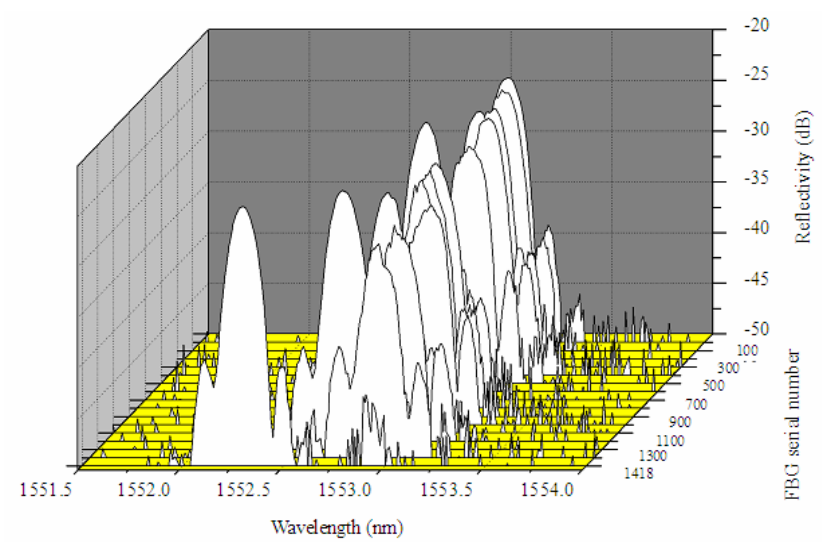

(b)

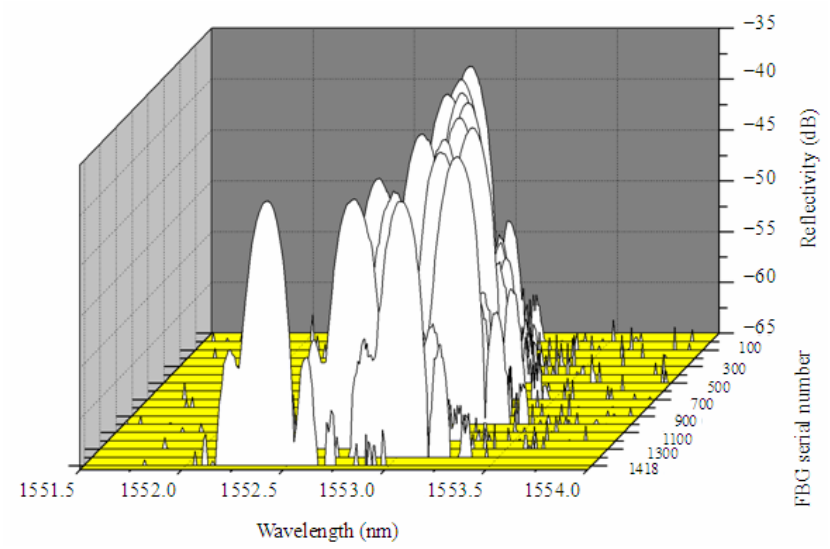

(c)

Fig. 18 Spectrum evolution of a 1418-DTG array: (a) fluctuant distribution of the peak wavelengths of a 1418-DTGs array, (b) reflection spectra of the 16 representative DTGs with a average reflectivity of $-24 \mathrm{~dB}$ and (c) reflection spectra of the 16 representative DTGs with an average reflectivity of $-38 \mathrm{~dB}$

A further distributed temperature measurement is conducted to investigate the sensing performance of the network made up of 6108 DTGs with an average reflectivity of $-38.5 \mathrm{~dB}$ in an about $10 \mathrm{~km}$ fiber. In order to depress notorious crosstalks in the large-capacity sensing array, the two wavelength bands of $1552.1 \mathrm{~nm}$ and $1552.6 \mathrm{~nm}$ were applied for it, and 3010 DTGs with a length of $6140 \mathrm{~m}$ and a neighboring DTG space of $2 \mathrm{~m}$ were in the $1552.6 \mathrm{~nm}$ band, and in the $1552.1 \mathrm{~nm}$ there were 3098 DTGs with a length of $3400 \mathrm{~m}$ and a neighboring DTG space of $1 \mathrm{~m}$. The sensing fiber was segmented into six sections, and they were winded on five collection drums, in which $4 \#$ and $6 \#$ sections were winded on one same drum. The sections $1 \#, 3 \#$, and $5 \#$ of the sensor array were heated in a temperature test chamber (SIDA TEMI300), while the rest of the DTG sensors were kept at room temperature of $25{ }^{\circ} \mathrm{C}$ (shown in Fig. 19(a)). The chamber temperature was stepped by $10{ }^{\circ} \mathrm{C}$ from $25{ }^{\circ} \mathrm{C}$ to $75{ }^{\circ} \mathrm{C}$ with an accuracy of $0.1{ }^{\circ} \mathrm{C}$. The result of temperature measurement is shown in Fig. 19(b). For the heated DTGs, their peak wavelengths showed red shift with an increase in temperature, and the two representative DTGs located at $242 \mathrm{~m}$ and $9000 \mathrm{~m}$ have a good linear response with a coefficient of $10.68 \mathrm{pm} /{ }^{\circ} \mathrm{C}$ [Fig. 19(c)]. 


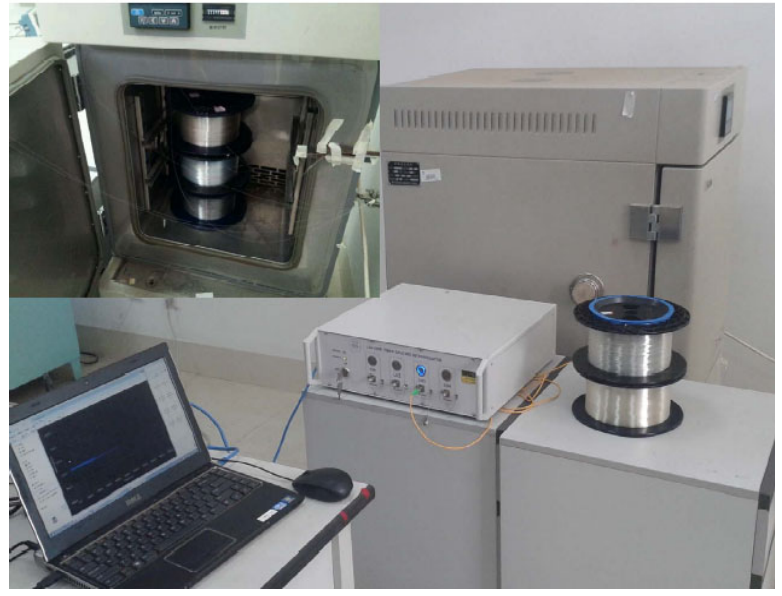

(a)

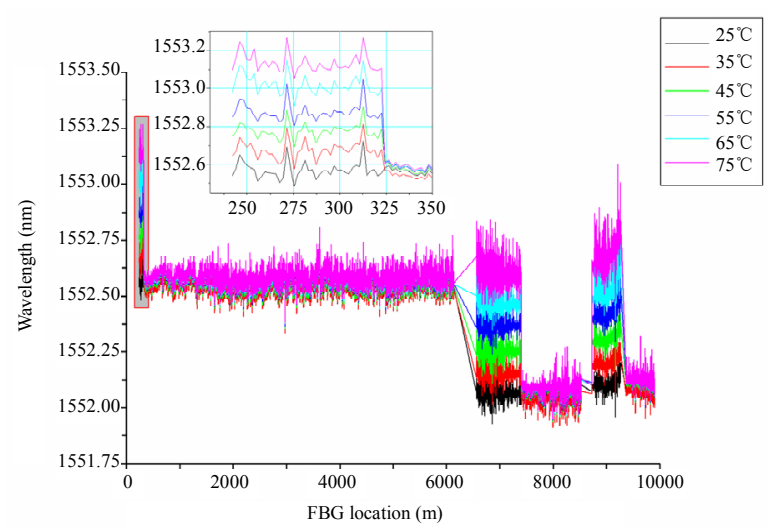

(b)

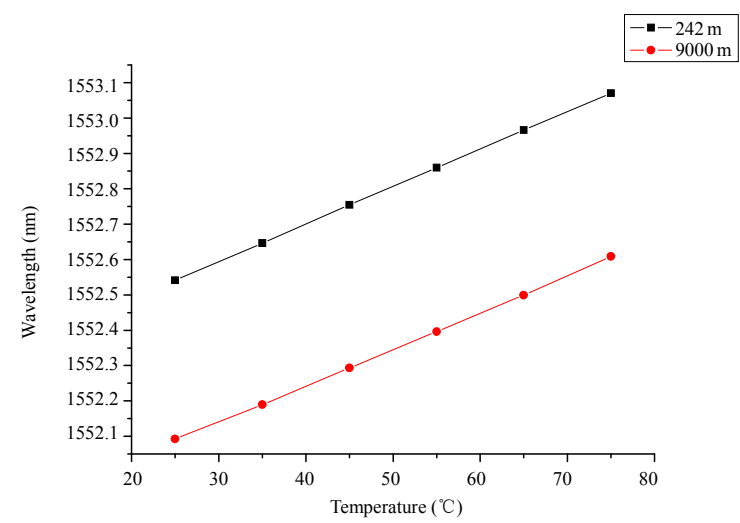

(c)

Fig. 19 Experimental demonstration: (a) images of the interrogation system of an ultra-weak DTG array, (b) temperature sensing performance of the 6018 ultra-weak DTG array, and (c) the static temperature response of the two representative DTGs located $242 \mathrm{~m}$ and $9000 \mathrm{~m}$.

\section{Conclusions}

As conclusion, this paper reviews the work on fiber-optic sensing network with huge capacity based on ultra-weak draw tower gratings developed at the National Engineering Laboratory for Fiber Optic Sensing Technology (NEL-FOST), Wuhan University of Technology, China. The draw-tower FBG arrays were prepared based on the phase mask technique, and the uniformity of the obtained FBG arrays was investigated. It was found that the uniformity of draw-tower FBG arrays greatly depended on the stability of the drawing process, especially the drawing tension. Under an optimized condition (the drawing speed: $12 \mathrm{~m} / \mathrm{min}$, the average drawing tension: 38.2 grams), one 300-FBG array with the average reflectivity of about $0.26 \%$ was prepared. They showed central wavelength bandwidth less than $0.1 \mathrm{~nm}$, the mean reflection power with the relative deviation of $\pm 16 \%$, and excellent overlapping of the peak shape. These indicated that on-line writing FBG array by the phase mask technique had a good uniformity of central wavelength. However, due to the unstable laser pulse energy, the uneven distribution of the writing energy density and the optical fiber vibration, the uniformity of reflection power was not perfect.

A novel interrogation system for large scale sensing network with identical ultra-weak FBGs system based on serial TDM scheme was proposed and experimentally demonstrated in this paper. 1009 FBGs with reflectivity of about $-33 \mathrm{~dB}$ and 2.5 meters equidistance between neighbor FBGs were multiplexed in series. The measurement accuracy of the Bragg wavelengths obtained by the interrogation system was less than $5 \mathrm{pm}$, and one round interrogation was completed within several seconds. The spectral distortion induced by multiple reflection crosstalks was measured and analyzed. The reflective spectra of FBGs in the end of the array showed a low transmission loss which can be further reduced by using even weaker FBGs, and this means the interrogation system has the ability to demodulate even larger number of sensors in serial.

Fiber-optic sensing network with huge capacity based on ultra-weak draw tower gratings is 
experimentally demonstrated as a new platform for optical fiber sensing technologies. The ultra-weak grating showed FWHM less than $0.1 \mathrm{~nm}$ with excellent overlapping of the peak fringe. Based on the developed ultra-weak FBG array, a versatile large-scale ultra-weak FBG sensor network with two SOAs and one high-speed CCD module has been proposed and demonstrated. Results show that the proposed sensor network can interrogate the large-scale array over 6000 FBGs by TDM+WDM. The pioneering design has low crosstalks and good sensing characteristic, and would be very promising for fiber optic sensing application.

\section{Acknowledgment}

This work was supported by the Major Program of the National Natural Science Foundation of China, NSFC (Grant No. 61290311) and the Natural Science Foundation of Hubei Province, China (No. 2014CFB269).

Open Access This article is distributed under the terms of the Creative Commons Attribution 4.0 International License (http:// creativecommons. org/ licenses/by/4.0/), which permits unrestricted use, distribution, and reproduction in any medium, provided you give appropriate credit to the original author(s) and the source, provide a link to the Creative Commons license, and indicate if changes were made.

\section{References}

[1] Y. Rao, A. B. Ribeiro, D. A. Jackson, L. Zhang, and I. Bennion, "Simultaneous spatial, time and wavelength division multiplexed in-fibre grating sensing network," Optics Communications, 1996, 125(1-3): 53-58.

[2] W. Ecke, I. Latka, R. Willsch, A. Reutlinger, and R. Graue, "Fibre optic sensor network for spacecraft health monitoring," Measurement Science and Technology, 2001, 12(7): 974-980.

[3] B. A. Childers, M. E. Froggatt, S. G. Allison, T. C. Moore, D. A. Hare, C. F. Batten, et al., "Use of 3000 Bragg grating strain sensors distributed on four 8-m optical fibers during static load tests of a composite structure," in Proc. SPIE, vol. 4332, pp. 133-142, 2001.

[4] D. J. F. Cooper, T. Coroy, and P. W. E. Smith,
"Time-division-multiplexing of large serial fiber-optic Bragg grating sensor arrays," Applied Optics, 2001, 40(16): 2643-2654.

[5] Y. Wang, J. Gong, D. Wang, B. Dong, W. Bi, and Anbo Wang, "A quasi-distributed sensing network with time-division-multiplexed fiber Bragg gratings," IEEE Photonics Technology Letters, 2011, 2(23): 70-72.

[6] Y. J. Rao, A. B. Lobo Ribeiro, D. A. Jackson, L. Zhang, and I. Bennion, "Simulataneous spatial, time and wavelength division multiplexed in- fiber grating sensing network," Optics Communications, 2012, 125: 53-58

[7] M. Zhang, Q. Sun, Z. Wang, X. Li, D. Liu, and H. Liu, "A large capacity sensing network with identical weak fiber Bragg gratings multiplexing," Optics Communications, 2012, 285(13): 3082-3087.

[8] W. Chung, H.Y. Tam, P. K. A. Wai, and A. Khandelwal, "Time- and wavelength-division multiplexing of FBG sensors using a semiconductor optical amplifier in ring cavity configuration," IEEE Photonics Technology letters, 2005, 17(12): 2709-2711.

[9] G. D. Lloyd, L. A. Everall, K. Sugden, and I. Bennion, "Resonant cavity time-division-multiplexed fiber Bragg grating sensor interrogator," IEEE Photonics Technology Letters, 2004, 16(10): 2323-2325.

[10] Y. Wang, J. Gong, B. Dong, D. Y. Wang, T. J. Shilig, and A. Wang, "A large serial time-division multiplexed fiber Bragg grating sensor network," Journal of Lightwave Technology, 2012, 30(17): 2751-2756.

[11] C. G. Askins, M. A. Putnam, H. J. Patrick, and F. J. Friebele, "Fiber strength unaffected by on-line writing of single-pulse Bragg gratings," Electronics Letters, 1997, 33(15): 1333-1334.

[12] V. Hagemann, M. N. Trutzel, L. Staudigel, M. Rothhardt, H. R. Muller, and O. Krumpholz, "Mechanical resistance of draw-tower-Bragg-grating sensors," Electronics Letters, 1998, 34(2): 211-212.

[13] M. Xu, H. Geiger, J. L. Archambault, L. Reekie, and J. P. Dakin, "Novel interrogating system for fiber Bragg grating sensors using an acousto-optic tunable filter," Electronics Letters, 1993, 29(17): 1510-1511.

[14] C. G. Askins, M. A. Putnam, G. M. Williams, and E. J. Friebele, "Stepped-wavelength optical-fiber Bragg grating arrays fabricated in line on a draw tower," Optics Letters, 1994, 19(2): 147-149.

[15] S. Abad, F. M. Araujo, L. A. Ferreira, J. L. Santos, and M. Lopez-Amo, "Comparative analysis of wavelength-multiplexed photonic-sensor networks using fused biconical WDMS," IEEE Sensors 
Journal, 2003, 3(4): 475-483.

[16] Y. Dai, Y. Liu, J. Leng, G. Deng, and A. Asundi, “A novel time-division multiplexing fiber Bragg grating sensor interrogator for structural health monitoring," Optics and Lasers in Engineering, 2009, 47(10): 1028-1033.

[17] X. Wan and H. F. Taylor, "Multiplexing of FBG sensors using modelocked wavelength-swept fibre laser," Electronics Letters, 2003, 39(21): 1512-1514.

[18] M. Zhang, Q. Sun, Z. Wang, X. Li, D. Liu, and H. Liu, "A large capacity sensing network with identical weak fiber Bragg gratings multiplexing," Optics Communications, 2012, 285(13-14): 3082-3087.

[19] B. A. Childers, M. E. Froggatt, S. G. Allison, T. C. Moore, D. A. Hare, C. F. Batten, and D. C. Jegley, "Use of 3000 Bragg grating strain sensors distributed on four 8-m optical fibers during static load tests of a composite structure," in Proc. SPIE, 2001, 4332(133), doi: 10.1117/12.429650.

[20] D. L. Williams, B. J. Ainslie, J. R. Armitage, and R. Kashyap, "Enhanced UV photosensitivity in boron codoped germanosilicate fibers," Electronics Letters, 1993, 29(1): 45-47.

[21] http://www.fbgs-technologies.com/pagina.php? $\mathrm{id}=21$
366

[22] B. Hartmut, S. Kay, U. Sonja, C. Christoph, R. Manfred, and L. Ines,“ Single-pulse fiber Bragg gratings and specific coatings for use at elevated temperatures," Applied Optics, 2007, 46(17): 3417-3424.

[23] D. L. Williams, B. J. Ainslie, R. Kashyap, G. D. Maxwell, J. R. Armitage, R. J. Campbell, et al., "Photosensitive index changes in germania-doped silica glass fibers and waveguides," in Proc. SPIE, vol. 2044, pp. 56-68, 1993.

[24] Y. Wang, J. Gong, D. Wang, B. Dong, W. Bi, and A. Wang, "A quasi-distributed sensing network with time-division-multiplexed fiber Bragg gratings," IEEE Photonics Technology Letters, 2011, 2(23): 70-72.

[25] C. Chan, W. Jin, D. Wang, and M. S. Demokan, "Intrinsic crosstalk analysis of a serial TDM FBG sensor array by using a tunable laser," Microwave and Optical Technology Letters, 2003, 36(1): 2-4.

[26] C. G. Askins, M. A. Putnam, and G. M. Williams, "Stepped-wavelength optical-fiber Bragg grating arrays fabricated in line on a draw tower," Optics Letters, 1994, 19(2): 147-149. 